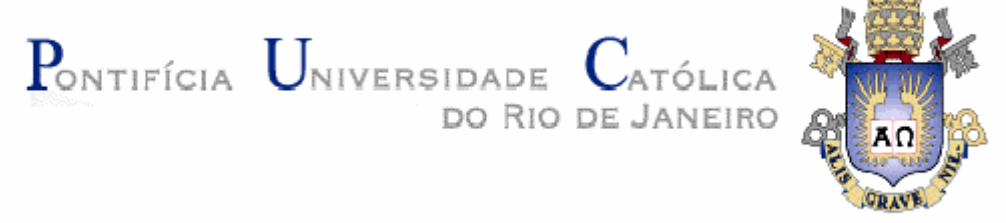

Tula Vieira Brasileiro

\title{
"Filho de": um estudo sobre o sub-registro de nascimento na cidade do Rio de Janeiro
}

Tese de Doutorado

Tese apresentada ao Programa de PósGraduação em Educação do Departamento de Educação da PUC-Rio como parte dos requisitos parciais para obtenção do título de Doutor em Educação.

Orientador: Profa. Tânia Dauster

Rio de Janeiro

Agosto de 2008 


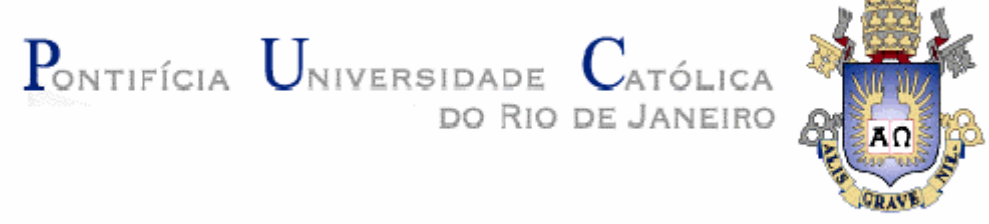

Tula Vieira Brasileiro

\section{"Filho de": um estudo sobre o sub-registro de nascimento na cidade do Rio de Janeiro}

Tese apresentada como requisito parcial para obtenção do título de Doutor pelo Programa de Pós-Graduação em Educação do Departamento de Educação do Centro de Teologia e Ciências Humanas da PUC-Rio. Aprovada pela Comissão Examinadora abaixo assinada.

Profa. Tânia Dauster M. e Silva Orientadora

Departamento de Educação - PUC-Rio

Profa. Sonia Kramer

Departamento de Educação - PUC-Rio

Prof $^{a}$. Irene Rizzini Departamento de Serviço Social - PUC-Rio

Prof $^{a}$. Myriam Moraes Lins de Barros

UFRJ

Prof. Luiz Cavalieri Bazilio

UERJ

Prof. Paulo Fernando C. de Andrade Coordenador Setorial do Centro de

Teologia e Ciências Humanas

Rio de Janeiro, 08 de agosto de 2008. 
Todos os direitos reservados. É proibida a reprodução total ou parcial do trabalho sem autorização da universidade, da autora e do orientador.

\section{Tula Vieira Brasileiro}

Tula Vieira Brasileiro, assistente social formada pela Universidade do Estado do Rio de Janeiro, UERJ, em 1988. Atuou por 12 anos na área da educação infantil, vinculada à Fundação Fé e Alegria do Brasil - RJ. Concluiu em 1995 o Curso de Especialização em Educação Infantil na Pontifícia Universidade Católica - PUC-Rio. Também pela PUC-Rio obteve o Mestrado e o Doutorado em Educação nos anos de 2001 e 2008 respectivamente. Atualmente trabalha como assistente social na Secretaria Estadual de Saúde, SES-RJ e na Secretaria Municipal de Assistência Social do Rio de Janeiro no Projeto Rede de Proteção ao Educando desenvolvido nas escolas do município do Rio de Janeiro.

Ficha Catalográfica

Brasileiro, Tula Vieira

"Filho de" : um estudo sobre o sub-registro de nascimento na cidade do Rio de Janeiro / Tula Vieira Brasileiro ; orientadora: Tânia Dauster. - 2008.

221 f. : II. ; $30 \mathrm{~cm}$

Tese (Doutorado em Educação)-Pontifícia Universidade Católica do Rio de Janeiro, Rio de Janeiro, 2008. Inclui bibliografia

1. Educação - Teses. 2. Sub-registro de nascimento. 3. Certidão de nascimento. 4. Infância. 5. Escolaridade. I. Dauster, Tânia. II. Pontifícia Universidade Católica do Rio de Janeiro. Departamento de Educação. III. Título. 
Dedico esta tese a todos os brasileiros que vivem a ausência da certidão nascimento na figura de Andréia.

$\mathrm{E}$ a todos os que lutam para que esta realidade seja modificada. 


\section{Agradecimentos}

Ao Lucas, meu filho, amor da minha vida;

À Tania Dauster, orientadora que acredita em mim;

À minha mãe, pelo amor de mãe;

Às minhas irmãs, irmãos, cunhadas e cunhado pelas infinitas ajudas;

A Inês, Teresa, Raquel e Ana Claudia, mães de amigos de Lucas por todas as colaborações;

Às amigas, Eliane e Dani, pela presença nas várias batalhas da vida;

Às amigas, Vânia e Monica, por sempre me acompanharem;

Às amigas do Hospital Estadual Carlos Chagas, em especial, Monica, Conceição, Elo, Rita, Neuri, Silvana e Liony por todas as ajudas ao longo desses anos;

Aos colegas da Prefeitura da Cidade do Rio de Janeiro por sempre tentarem me ajudar;

À Ana Paula pela transcrição das fitas;

À Silvia e Leila, pelo carinho com meu filho e retaguarda da casa;

À Carla e Adriani, amigas de sempre;

À Michele, pela preciosa colaboração ao longo do trabalho de campo;

À Maria do Carmo, pela confecção do heredograma;

A SES/RJ e ao Centro de Estudos do HECC, pela possibilidade de realizar o trabalho de campo no hospital;

A Simone, pela gentileza em me abrir as portas do Projeto Cidadania;

Aos professores Sonia Kramer, Irene Rizzini, Myriam Lins de Barros e Luiz Bazílio por comporem minha banca.

A todas as pessoas que se dispuseram a fornecer informações e histórias para essa pesquisa. 


\section{Resumo}

Brasileiro, Tula Vieira; Dauster, Tania. " "Filho de": um estudo sobre o sub-registro de nascimento na cidade do Rio de Janeiro.” Rio de Janeiro, 2008, 221 p. Tese de Doutorado - Departamento de Educação, Pontifícia Universidade Católica do Rio de Janeiro.

Esta tese nasceu da minha inserção profissional como assistente social em uma unidade de saúde de emergência do Estado do Rio de Janeiro - Hospital Estadual Carlos Chagas - HECC, onde encontrei várias crianças que não possuíam sua certidão de nascimento. $\mathrm{O}$ estranhamento que me causou este fato tornou-se maior ao perceber recorrências significativas desta situação e suas reverberações. Verifiquei que essas crianças eram chamadas pelos profissionais do HECC como "Filho de" acrescido do nome da mãe. Aos poucos compreendi que a ausência de certidão de nascimento se constitui em um fenômeno oficialmente denominado sub-registro de nascimento, a respeito do qual há pouca produção teórica, como pude verificar ao longo do processo de investigação. Tal problemática veio a ser a minha questão de pesquisa. Meu objetivo nesta tese foi entender porque algumas crianças atendidas no HECC, no período compreendido entre os anos de 1999 a 2007, não foram registradas. Buscando uma abordagem etnográfica, procurei desvendar as razões deste fato. Para tanto investiguei os significados da certidão de nascimento com mães e pais e suas implicações nas interações com as instituições escolares. Já que algumas crianças não foram registradas porque suas mães também não o foram, incorporei no processo de investigação adultos igualmente sem certidão de nascimento. Tal problemática me sugere uma questão instigante para o debate sobre o registro civil que envolve a reprodução social dos setores pobres da sociedade. Com este estudo, pretendo contribuir para a compreensão da história da infância e da família pobre brasileira, na expectativa de que esta investigação seja um alerta para os setores responsáveis pelo sistema de registro civil no Brasil. Ademais, pretendo contribuir para a elaboração de políticas públicas educacionais. Como instrumentos de trabalho, utilizei um questionário, entrevistas abertas, conversas com mães e pais, observações, documentos e material institucional do HECC. Meu diálogo central se deu com Roberto DaMatta (1997a; 1997; 2000), Ana Liési Thurler (2004); Cynthia Sarti (1996;2004) e Cláudia Fonseca (2005;no prelo).

\section{Palavras-chave:}

escolaridade.

Sub-registro de nascimento; certidão de nascimento; infância; 


\section{Abstract}

Brasileiro, Tula Vieira; Dauster, Tania (Advisor). “ “Child of”: a study of birth under-registration in Rio de Janeiro.” Rio de Janeiro, 2008, 221 p. Thesis - Departamento de Educação, Pontifícia Universidade Católica do Rio de Janeiro.

This paper is a consequence of my professional insertion as a social assistant in an emergency room unity of Rio de Janeiro State - Hospital Estadual Carlos Chagas - HECC, where I met several children who did not have a birth certificate. This fact surprised me even more when I realized the situation was significantly recurrent, as well as its reverberations. I noticed that these children were referenced by HECC staff as "Child of", plus the name of their mothers. Slowly I understood that the birth certificate absence constitutes a phenomenon officially called birth under-registration, about what there is a few theoric production, as I could verify during the investigation process. This issue is my research's subject. My goal with this paper was to understand why some children treated in HECC during the period between 1999 and 2007 were not registered. Seeking for an ethnographic approach, my objective was to find out the reasons for that fact. In order to do that, I have investigated the meanings of birth certificates with mothers and fathers, and their implication on interactions with educational institutions. Since some children were not registered because their mothers were not registered neither, I have incorporated the adults with no birth certificate in the investigation process. This issue suggests me an intriguing question for the debate about civil register which involves a social reproduction of society poor sectors. With this paper, I intend to contribute for the understanding of childhood background and Brazilian's poor families, expecting that this investigation is an alert for the sectors which are responsible for the civil register system in Brazil. In addition, I intend to contribute for the elaboration of educational public policies. As working tools, I used a questionnaire, open interviews, conversations with mothers and fathers, documents and institutional material from HECC. My central dialogue occurred with Roberto DaMatta (1997a; 1997; 2000), Ana Liési Thurler (2004); and Cynthia Sarti (1996;2004) e Cláudia Fonseca (2005; in edition).

\section{Key-words:}

Birth under-registration; birth certificate; childhood; scholarship. 


\section{Sumário}

1 Introdução

2 As situações de contato no trabalho de campo: perfil sociológico das crianças e responsáveis .............................. 19

2.1 Caminhos metodológicos ......................................... 19

$2.2 \quad$ Perfil das crianças e responsáveis ........................................ 43

3 A certidão de nascimento ou quando existir depende de um

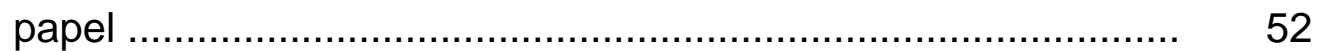

3.1 Sistema de registro civil no Brasil: alguns aspectos ................. 52

3.1.1 O que é a certidão de nascimento na perspectiva das mães e alguns pais ............................................................ 60

3.1.1.1 "Filho de" ........................................................... 73

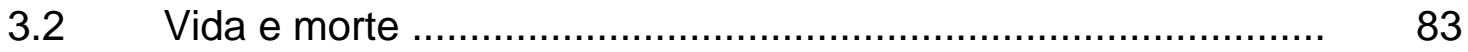

3.3 Ritual da certidão de nascimento ...................................... 88

3.3.1 Significados da escolha do nome dos filhos .......................... 101

$4 \quad$ Por quê não registrou seu filho? ......................................... 111

4.1 Porque as crianças não foram registradas .............................. 111

4.1.1 A espera pelo pai .................................................. 121

4.1.2 O mundo dos documentos .............................................. 143

4.1.3 Adultos sem certidão de nascimento .................................... 149

5 Conclusão - os horizontes constrangidos da escolaridade: considerações finais ............................................... 167

$6 \quad$ Referências bibliográficas ......................................... 181

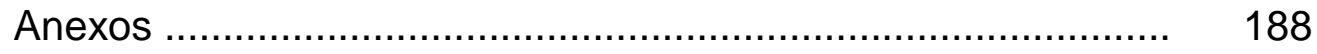




\section{Lista de Siglas}

ABEP - Associação Brasileira de Estudos Populacionais

ABA - Associação Brasileira de Antropologia

ANDI - Agência de Notícias dos Direitos da Infância

ANPED - Associação Nacional de Pós Graduação e Pesquisa em Educação

ANPOCS - Associação Nacional de Pós Graduação e Pesquisa em ciências sociais

BPC - Benefício de Prestação Continuada

CAPES - Coordenação de Aperfeiçoamento de Pessoal de Nível Superior

CIEP - Centro Integrado de Educação Pública

CDEDICA - Núcleo Especializado de Defesa dos Direitos da Criança e do Adolescente da Defensoria Pública do Estado do Rio de Janeiro

CRE - Coordenadoria de Educação

CPF - Cadastro de Pessoas Físicas

DETRAN - Departamento de Trânsito do Estado do Rio de Janeiro

DN - Declaração de Nascido Vivo

HECC - Hospital Estadual Carlos Chagas

IBGE - Instituto Brasileiro de Geografia e Estatística

IDS - Índice de Desenvolvimento Social

PBF - Programa Bolsa Família 
SEI - Serviço de Internação e Alta

SPA - Serviço de Pronto Atendimento

PBF - Programa Bolsa Família

SES - Secretaria Estadual de Saúde

SINASC - Sistema de Informações Sobre Nascidos Vivos

SMAS - Secretaria Municipal de Assistência Social

SME - Secretaria Municipal de Educação

ONU - Organização das Nações Unidas

UNICEF - Fundo das Nações Unidas Para a Infância 